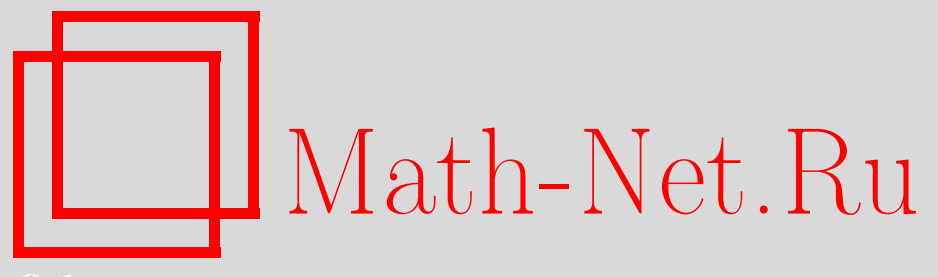

Ю. В. Таранников, О значениях аффинного ранга носителя спектра платовидной функции, Дискрет. матем., 2006, том 18, выпуск 3, 120-137

DOI: https://doi.org/10.4213/dm65

Использование Общероссийского математического портала Math-Net.Ru подразумевает, что вы прочитали и согласны с пользовательским соглашением http: //www . mathnet.ru/rus/agreement

Параметры загрузки:

IP: 34.227 .88 .159

26 апреля 2023 г., 11:55:00 


\title{
О значениях аффинного ранга носителя спектра платовидной функции
}

\author{
() 2006 г. Ю. В. Таранников
}

\begin{abstract}
В статье доказывается, что аффинный ранг любой платовидной функции с носителем спектра мощности 16 равен 4, 5 или 6. Для любого натурального $h$ рассматриваются платовидные функции с носителем спектра мощности $4^{h}$, даются оценки аффинного ранга таких функций и строятся функции, аффинный ранг которых принимает все возможные значения от $2 h$ до $2^{h+1}-2$.
\end{abstract}

\section{1. Введение и основные определения}

Пусть $F_{2}^{n}$ - векторное пространство наборов длины $n$ с компонентами из поля $F_{2}$ из двух элементов 0 и 1 , операции сложения и умножения в котором вводятся как обычные операции сложения и умножения чисел 0 и 1 по модулю 2. Булева функщия от $n$ переменных - это отображение из $F_{2}^{n}$ в $F_{2}$. В дальнейшем мы будем обозначать набор из $F_{2}^{n}$ буквой без нижнего индекса, а компоненту этого набора - той же буквой с нижним индексом, указываюшим на порядковый номер этой компоненты в наборе. Наборы $x^{\prime}$ и $x^{\prime \prime}$ называются соседними, если они различаются только в одной компоненте. Обозначим через $x^{i}$ набор, который отличается от $x$ только в $i$-й компоненте, $i=1, \ldots, n$. Переменная $x_{i}$ называется фиктивной для функция $f$, если для любых наборов $x^{\prime}$ и $x^{\prime \prime}$, соседних по $i$-й компоненте, выполняется равенство $f\left(x^{\prime}\right)=f\left(x^{\prime \prime}\right)$. Расстоянием Хэмминга $d\left(x^{\prime}, x^{\prime \prime}\right)$ между двумя наборами $x^{\prime}$ и $x^{\prime \prime}$ называют число компонент, в которых наборы $x^{\prime}$ и $x^{\prime \prime}$ различаются. Для заданной функщия $f$ из $F_{2}^{n}$ минимум расстояний $d(f, l)$, где $l$ пробегает множество всех аффинных функций из $F_{2}^{n}$ называется нелинейностью функции $f$ и обозначается через $\mathrm{nl}(f)$. Подфункцией булевой функции $f$ называется функция $f^{\prime}$, полученная подстановкой в $f$ констант 0 или 1 вместо некоторых переменных.

Хорошо известно, что функция $f$, заданная на $F_{2}^{n}$, имеет единственное полиномиальное представление над $F_{2}$, степень которого по каждой переменной не превосходит 1 , a именно,

$$
f\left(x_{1}, \ldots, x_{n}\right)=\bigoplus_{\left(a_{1}, \ldots, a_{n}\right) \in F_{2}^{n}} g\left(a_{1}, \ldots, a_{n}\right) x_{1}^{a_{1}} \ldots x_{n}^{a_{n}},
$$

где $g$ - также функция над $F_{2}^{n}$. Такое полиномиальное представление $f$ называется алгебраической нормальной формой функции, а каждое выражение $x_{1}^{a_{1}} \ldots x_{n}^{a_{n}}$ называется слагаемым в алгебраической нормальной форме функции $f$ (алгебраическую нормальную форму называют также полиномом Жегалкина). Отображение $f(x) \rightarrow g(x)$ иногда называют преобразованием Мебиуса. 
Алгебраическая степень функции $f$, обозначаемая через $\operatorname{deg}(f)$, определяется как число переменных в самом длинном слагаемом в алгебраической нормальной форме функции $f$.

$\operatorname{Bec} \operatorname{wt}(f)$ функции $f$ над $F_{2}^{n}$ - это число наборов $x$ из $F_{2}^{n}$, для которых $f(x)=1$. Функщия $f$ называется уравновешенной, если

$$
\operatorname{wt}(f)=\operatorname{wt}(f \oplus 1)=2^{n-1},
$$

то есть если функция принимает значения 0 и 1 на одинаковом числе наборов.

Пусть $x=\left(x_{1}, \ldots, x_{n}\right)$ и $u=\left(u_{1}, \ldots, u_{n}\right)$ - это наборы длины $n$ над $F_{2}$. Скалярное произведение $x$ и $u$ - это функция, которая определяется как

$$
\langle x, u\rangle=\sum_{i=1}^{n} x_{i} u_{i},
$$

где сложение и умножение берутся над $F_{2}$. Под суммой $x+u$ двух двоичных наборов $x$ и $u$ мы понимаем их покомпонентную сумму над $F_{2}$.

Преобразованием Уолша булевой функции $f$ называется целочисленная функция над $F_{2}^{n}$, определяемая равенством

$$
W_{f}(u)=\sum_{x \in F_{2}^{n}}(-1)^{f(x)+\langle u, x\rangle} .
$$

Для каждого $u \in F_{2}^{n}$ значение $W_{f}(u)$ называется коэффициентом Уолша. Коэффициенты Уолша будем называть спектральными коэффициентами, а совокупность всех $2^{n}$ коэффициентов Уолша - спектром булевой функции. Справедливы формула обращения

$$
(-1)^{f(x)}=2^{-n} \sum_{u \in F_{2}^{n}} W_{f}(u)(-1)^{\langle u, x\rangle}
$$

и равенство Парсеваля

$$
\sum_{u \in F_{2}^{n}} W_{f}^{2}(u)=2^{2 n}
$$

Нелинейность булевой функции $f$ выражается через ее коэффициенты Уолша следующим образом:

$$
\mathrm{nl}(f)=2^{n-1}-\frac{1}{2} \max _{u \in F_{2}^{n}}\left|W_{f}(u)\right| .
$$

Множество $S_{f}$ всех наборов $u$ таких, что $W_{f}(u) \neq 0$, называется носителем спектра функции $f$.

Булева функщия называется бент-функщией, если значение коэффициентов на всех наборах равно $\pm 2^{n / 2}$. Бент-функции существуют при всех четных $n$, а при нечетных не существуют. Бент-функция является функцией с максимально возможной нелинейностью $2^{n-1}-2^{(n / 2)-1}$ среди всех функций от $n$ переменных при четном $n$. Булева функция называется платовидной, если ее коэффициенты Уолша принимают ровно три возможных значения. Несложно показать, что эти значения могут быть только 0 и $\pm 2^{c}$ для некоторого $c$. Платовидные функции представляют большой интерес для изучения бент-функщй (например, потому, что при разложении бент-функции по переменной возникают две платовидные функции), а также потому, что многие криптографически важные функции являются платовидными (например, $m$-устойчивые функщии с максимально возможной для 
них нелинейностью $\left.2^{n-1}-2^{m+1}\right)$. Для платовидных функций положим $\varphi(x)=2^{-c} W_{f}(x)$, тогда для любого $x \in F_{2}^{n}$ величина $\varphi(x)$ может принимать только три значения: $0,-1$ и 1 . Множество $S_{f}$ всех наборов $u$ таких, что $W_{f}(u) \neq 0$, называется носителем спектра платовидной функции. Множество всех наборов, на которых $\varphi(x)=-1$, будем обозначать $T^{-}$, а множество всех наборов, на которых $\varphi(x)=1$, будем обозначать $T^{+}$. Из равенства Парсеваля следует, что мощность носителя спектра равна $4^{n-c}$. Бент-функцию удобно рассматривать как частный случай платовидной при $c=n / 2$ и $\left|S_{f}\right|=2^{n}$, что мы и будем часто делать (хотя часто формально бент-функции к платовидным не относят). Платовидные функции изучались в большом числе работ, см., например, $[1,4,9]$.

Для каждого $u \in F_{2}{ }^{n}$ автокорреляционный коэффициент функции $f$ на наборе $u$ определяется как

$$
\Delta_{f}(u)=\sum_{x \in F_{2}{ }^{n}}(-1)^{f(x)+f(x+u)} .
$$

Функция $D_{u} f=f(x)+f(x+u)$ называется производной функции $f$ по направлению $u$. Множество наборов $u \in F_{2}{ }^{n}$ таких, что $D_{u} f$ есть постоянная, называется линейной структурой функции $f$. Легко проверить, что линейная структура функции $f$ образует линейное пространство в $F_{2}^{n}$. Наличие у функции линейных структур в некоторых случаях (но не во всех) является криптографической слабостью.

Пусть $E$ - произвольное подмножество $F_{2}^{n}$. Рангом множества $E$ называется размерность подпространства, порожденного $E$ в $F_{2}^{n}$. Аффинным рангом множества $E$ называется размерность наименьшего класса смежности в $F_{2}^{n}$, содержащего $E$. Ранг и аффинный ранг носителя спектра булевой функции будем обозначать через $k$ и $k_{a}$, соответственно. Для краткости в данной работе аффинный ранг и ранг носителя спектра булевой функция мы будем называть просто ее аффинным рангом и рангом, соответственно. Легко убедиться, что $k_{a} \in\{k, k-1\}$. Известно (см., например, [5]), что размерность линейной структуры функции $f$ равна $n-k_{a}$. Если существует набор $u \in F_{2}^{n}$ такой, что $D_{u} f \equiv 1$, то $k=k_{a}+1$. Если такого набора не сушествует, то $k=k_{a}$.

Дополнительные сведения о свойствах булевых функщий можно найти в [2] и [3].

\section{2. Об аффинных преобразованиях в $F_{2}^{n}$}

Аффинным преобразованием в $F_{2}^{n}$ называется отображение

$$
x \rightarrow x^{\prime}=\mathbf{A} x=x A^{T}+a,
$$

где $A$ - квадратная двоичная невырожденная над $F_{2}$ матрица порядка $n$, а $a$ - вектор длины $n$. Аффинное преобразование является автоморфизмом $F_{2}^{n}$, при котором все классы смежности переходят в классы смежности той же размерности. Если $a=0$, то аффинное преобразование называется также линейным.

Аффинным преобразованием функции $f$, заданной на $F_{2}^{n}$, называется преобразование

$$
f(x) \rightarrow f^{\prime}(x)=f(\mathbf{A} x) .
$$

Если для функций $f$ и $f^{\prime}$ существует аффинное преобразование функции, переводящее $f$ в $f^{\prime}$, то $f$ и $f^{\prime}$ называются аффинно эквивалентными. Если для функций $f$ и $f^{\prime}$ существует линейное преобразование функции, переводящее $f$ в $f^{\prime}$, то $f$ и $f^{\prime}$ называются линейно эквивалентными. 
Лемма 1. Пусть $f(x) \rightarrow f^{\prime}(x)=f(\mathbf{A} x)$ - аффинное преобразование функции $f$, заданной на $F_{2}^{n}$. Тогда

$$
W_{f^{\prime}(x)}(u)=(-1)^{\left(a, u A^{-1}\right\rangle} W_{f}\left(u A^{-1}\right) .
$$

Доказательство. По формуле для коэффициентов Уолша

$$
\begin{aligned}
W_{f^{\prime}(x)}(u) & =\sum_{x \in F_{2}^{n}}(-1)^{f^{\prime}(x)+\langle x, u\rangle}=\sum_{x \in F_{2}^{n}}(-1)^{f(\mathrm{~A} x)+\langle x, u\rangle}=\sum_{x \in F_{2}^{n}}(-1)^{f(x)+\left\langle\mathrm{A}^{-1} x, u\right\rangle} \\
& =\sum_{x \in F_{2}^{n}}(-1)^{f(x)+\left\langle x, u A^{-1}\right\rangle+\left\langle a, u A^{-1}\right\rangle}=(-1)^{\left\langle a, u A^{-1}\right\rangle} \sum_{x \in F_{2}^{n}}(-1)^{f(x)+\left\langle x, u A^{-1}\right\rangle} \\
& =(-1)^{\left\langle a, u A^{-1}\right\rangle} W_{f}\left(u A^{-1}\right) .
\end{aligned}
$$

Пусть булева функция $f$ задана на $F_{2}^{n}$. Аффинным преобразованием спектра функции $f$ называется преобразование

$$
W_{f}(x) \rightarrow W^{\prime}(x)=W_{f}(\mathbf{A} x) .
$$

Можно показать, что коэффициенты $W^{\prime}(x)$ являются коэффициентами Уолша некоторой функщия $f^{\prime}$, которая, вообще говоря, не является аффинно эквивалентной функции $f$.

Лемма 2. Пусть $W_{f}(x) \rightarrow W^{\prime}(x)=W_{f}(\mathbf{A} x)$ - аффинное преобразование спектра функчии $f$, заданной на $F_{2}^{n}$. Тогда коэффичиенты $W^{\prime}(x)$ являются коэффичиентами Уолша некоторой функчии $f^{\prime}$, причем

$$
f^{\prime}(x)=f\left(x A^{-1}\right)+\left\langle a, x A^{-1}\right\rangle .
$$

Доказательство. Проверим, что для всех $x \in F_{2}^{n}$ суммы в формуле обращения для гипотетически существующей функции $f^{\prime}(x)$ равны \pm 1 . Введем обозначение

$$
F(x)=2^{-n} \sum_{u \in F_{2}^{n}} W^{\prime}(u)(-1)^{\langle u, x\rangle} .
$$

\section{Справедливы равенства}

$$
\begin{aligned}
F(x) & =2^{-n} \sum_{u \in F_{2}^{n}} W^{\prime}(u)(-1)^{\langle u, x\rangle}=2^{-n} \sum_{u \in F_{2}^{n}} W_{f}(\mathbf{A} u)(-1)^{\langle u, x\rangle} \\
& =2^{-n} \sum_{v \in F_{2}^{n}} W_{f}(v)(-1)^{\left\langle\mathbf{A}^{-1} v, x\right\rangle}=2^{-n} \sum_{v \in F_{2}^{n}} W_{f}(v)(-1)^{\left\langle v, x A^{-1}\right\rangle+\left\langle a, x A^{-1}\right\rangle} \\
& =(-1)^{\left\langle a, x A^{-1}\right\rangle} 2^{-n} \sum_{v \in F_{2}^{n}} W_{f}(v)(-1)^{\left\langle v, x A^{-1}\right\rangle}=(-1)^{f\left(x A^{-1}\right)+\left\langle a, x A^{-1}\right\rangle} .
\end{aligned}
$$

Таким образом, для всех $x \in F_{2}^{n}$ справедливо равенство $F(x)= \pm 1$. Поэтому функция $f^{\prime}(x)$ существует, более того,

$$
f^{\prime}(x)=f\left(x A^{-1}\right)+\left\langle a, x A^{-1}\right\rangle .
$$

Спектры функций $f$ и $f^{\prime}$, переводимые один в другой аффинным преобразованием спектра, называются аффинно эквивалентными. Спектры функщий $f$ и $f^{\prime}$, переводимые 
один в другой линейным преобразованием спектра, называются линейно эквивалентными. Из аффинной эквивалентности функщий не следует аффинная эквивалентность их спектров. Например, потому, что при аффинном преобразовании функции $f$ величина $\operatorname{wt}(f)$ остается неизменной, но $\operatorname{wt}(f)=2^{n-1}-(1 / 2) W_{f}(0)$, поэтому переводя при аффинном преобразовании спектра в 0 набор с другим значением коэффициента Уолша, мы получим функцию, не являющуюся аффинно эквивалентной $f$. В то же время из лемм 1 и 2 видно, что линейное преобразование спектра является линейным преобразованием функции, и наоборот.

Очевидно, что аффинное преобразование спектра платовидной функции $f$ переводит его в спектр также некоторой платовидной функции $f^{\prime}$ с той же мощностью носителя спектра, а аффинное преобразование платовидной функции $f$ переводит ее в платовидную функцию $f^{\prime}$ с той же мощностью носителя спектра.

Лемма 3. Пусть $f$ - булева функчия, заданная на $F_{2}^{n}$, причем носитель спектра этой функиии лежит в $F_{2}^{l} \otimes(0, \ldots, 0)$, где $(0, \ldots, 0) \in F_{2}^{n-l}$. Тогда функиия $f$ зависит от переменных $x_{l+1}, \ldots, x_{n}$ фиктивно.

Пусть $f^{\prime}-$ функиия на $F_{2}^{n}$, полученная из $f$ удалением фиктивных переменных $x_{l+1}, \ldots, x_{n}$. Тогда для любого и из $F_{2}^{l}$ справедливо равенство

$$
W_{f^{\prime}}(u)=2^{-(n-l)} W_{f}(u, 0, \ldots, 0),
$$

где $(u, 0, \ldots, 0) \in F_{2}^{n}$.

Доказательство. Пусть $x$ и $x^{i}$ - произвольная пара наборов, соседних по $i$-й компоненте, $i \in\{l+1, \ldots, n\}$. По формуле обращения

$$
\begin{aligned}
(-1)^{f(x)}-(-1)^{f\left(x^{i}\right)} & =2^{-n} \sum_{u \in F_{2}^{n}} W_{f}(u)\left((-1)^{\langle x, u\rangle}-(-1)^{\left\langle x^{i}, u\right\rangle}\right) \\
& =2^{-n} \sum_{u \in F_{2}^{l} \otimes(0, \ldots, 0)} W_{f}(u)\left((-1)^{\langle x, u\rangle}-(-1)^{\left\langle x^{i}, u\right\rangle}\right)=0 .
\end{aligned}
$$

Поэтому $f(x)=f\left(x^{i}\right)$, и переменные $x_{l+1}, \ldots, x_{n}$, таким образом, действительно являются фиктивными. Рассмотрим теперь функцию $f^{\prime}$ на $F_{2}^{n}$, полученную из $f$ удалением фиктивных переменных $x_{l+1}, \ldots, x_{n}$. Для любого ее коэффициента Уолша

$$
\begin{aligned}
W_{f^{\prime}}(u) & =\sum_{x \in F_{2}^{l}}(-1)^{f^{\prime}(x)+\langle x, u\rangle}=2^{-(n-l)} \sum_{x \in F_{2}^{n}}(-1)^{f(x)+\langle x,(u, 0, \ldots, 0)\rangle} \\
& =2^{-(n-l)} W_{f}(u, 0, \ldots, 0),
\end{aligned}
$$

где $u \in F_{2}^{l}$ и $(u, 0, \ldots, 0) \in F_{2}^{n}$.

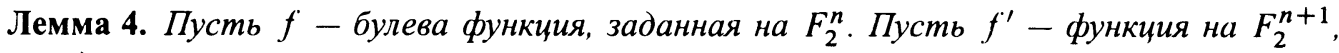
определенная равенством

$$
f^{\prime}\left(x_{1}, \ldots, x_{n}, x_{n+1}\right)=f\left(x_{1}, \ldots, x_{n}\right)+x_{n+1} .
$$

Тогда если и - набор из $F_{2}^{n+1}$, принадлежащий носителю спектра функиии $f^{\prime}$, то

$$
u_{n+1}=1, \quad W_{f^{\prime}}\left(u_{1}, \ldots, u_{n}, 1\right)=2 W_{f}\left(u_{1}, \ldots, u_{n}\right) \text {. }
$$


Доказательство. Пусть $u \in F_{2}^{n+1}$. В сумме

$$
W_{f^{\prime}}(u)=\sum_{x \in F_{2}^{n+1}}(-1)^{f(x)+\langle x, u\rangle}
$$

сгруппируем в пары наборы $x$ и $x^{n+1}$, различающиеся только в $(n+1)$-й компоненте. Для определенности пусть $x_{n+1}=0$. Для этих наборов $f^{\prime}(x)=f^{\prime}\left(x^{n+1}\right)+1$. Если $u_{n+1}=0$, то $\langle x, u\rangle=\left\langle x^{n+1}, u\right\rangle$. Отсюда получаем, что

$$
(-1)^{f^{\prime}(x)+\langle x, u\rangle}+(-1)^{f^{\prime}\left(x^{n+1}\right)+\left\langle x^{n+1}, u\right\rangle}=0 .
$$

Поэтому и $W_{f^{\prime}}(u)=0$. Если $u_{n+1}=1$, то $\langle x, u\rangle=\left\langle x^{n+1}, u\right\rangle+1$. Отсюда получаем, что

$$
\begin{aligned}
(-1)^{f^{\prime}(x)+\langle x, u\rangle}+(-1)^{f^{\prime}\left(x^{n+1}\right)+\left\langle x^{n+1}, u\right\rangle} & =2(-1)^{f^{\prime}(x)+\langle x, u\rangle} \\
& =2(-1)^{f\left(x_{1}, \ldots, x_{n}\right)+\left(\left(x_{1}, \ldots, x_{n}\right),\left(u_{1}, \ldots, u_{n}\right)\right\rangle} .
\end{aligned}
$$

Поэтому $W_{f^{\prime}}(u)=2 W_{f}\left(u_{1}, \ldots, u_{n}\right)$.

Из лемм 1, 2 и 3 следует, что изучение платовидных функций на $F_{2}^{n}$ с носителем спектра мощности $4^{h}$ можно в некотором смысле свести к изучению платовидных функщий с носителем спектра той же мощности $4^{h}$, заданных на $F_{2}^{k_{a}}$. Более того, если $k_{a}>2 h$, то любую платовидную функцию $f^{\prime}$ на $F_{2}^{n}$ с носителем мощности $4^{h}$ можно получить из некоторой функции $f$ на $F_{2}^{k_{a}}$ с носителем той же мощности $4^{h}$, добавив $n-k_{a}$ фиктивных переменных и выполнив некоторое линейное преобразование функции. Того же можно добиться и в случае, если $k_{a}=2 h$ и $W_{f^{\prime}}(0) \neq 0$ (в этом случае функция $f$ будет бент-функцией). Если $k_{a}=2 h$ и $W_{f^{\prime}}(0)=0$, то указанного линейного преобразования функции не существует, но можно использовать аффинное преобразование спектра, либо же взять функцию $f$ от $k_{a}+1$ переменной.

Заметим, что указанного сведения может быть недостаточно, если требуется исследовать дополнительные свойства функщий, не сохраняющиеся при аффинных преобразованиях (например, корреляционную иммунность).

Укажем также на следующее свойство, позволяющее не рассматривать специально вопрос о возможных значениях, принимаемых рангом $k$.

Лемма 5. Если существует платовидная функция с носителем спектра мощности $4^{h}$ и аффинным рангом, равным $k_{a}$, то существуют платовидные функиии $с$ носителем спектра той же мочности $4^{h}$ и рангами, равными и $k=k_{a}$, и $k=k_{a}+1$.

Доказательство. По лемме 2 аффинное преобразование носителя спектра платовидной функции снова дает платовидную функцию с носителем спектра той же мощности и с тем же аффинным рангом. Если аффинным преобразованием перевести в ноль один из наборов, входящий в наименьший класс смежности, содержащий $S_{f}$, то для получившейся функции, очевидно, будет выполнено равенство $k=k_{a}$. Если же перевести в ноль набор, не принадлежащий наименьшему классу смежности, содержащему $S_{f}$, то для получившейся функции $k=k_{a}+1$. Если для исходной функции не было наборов, не принадлежащих наименьшему классу смежности, содержащему $S_{f}$ (то есть, если $k_{a}$ совпадало с числом переменных), то можно просто добавить фиктивную переменную и такие наборы появятся, а функция останется платовиднои́ с той же мощностью спектра. 
Из вышесказанного следует, что аффинный ранг является важной характеристикой платовидных функщий. Очевидно, что аффинный ранг любой платовидной функщии с носителем спектра мощности $4^{h}$ не меньше $2 h$, потому что меньшие классы смежности не содержат $4^{h}$ наборов. Любая платовидная функция с носителем спектра мощности 1 есть аффинная функщия и, очевидно, ее аффинный ранг равен 0. Аффинный ранг любой платовидной функщии с носителем спектра мощности 4 равен 2. Этот факт доказан в [8], но, по-видимому, был известен намного раньше. Платовидные функции с носителем спектра мощности 16, не называясь платовидными, фактически рассматривались в [7], а в [5] для подкласса платовидных функщий с носителем спектра мощности 16 (более точно, для кубических устойчивых порядка $n-4$ функций) была получена оценка $k_{a} \leqslant k \leqslant 9$. В настоящей работе мы докажем, что аффинный ранг любой платовидной функции с носителем спектра мощности 16 равен 4, 5 или 6. Кроме того, мы рассмотрим для любого натурального $h$ платовидные функции с носителем спектра мощности $4^{h}$, дадим оценки аффинного ранга для таких функщий и построим функщии, аффинный ранг которых принимает все возможные значения от $2 h$ до $2^{h+1}-2$.

\section{3. Вспомогательные результаты}

Следующее утверждение хорошо известно (см., например, соотношение (2.16) в [2]). Ранее его доказательство было дано в [6].

Лемма 6. Пусть $f-$ булева функция на $F_{2}^{n}$. Пусть $U$ является линейным подпространством в $F_{2}^{n}$ размерности $l$, a $U^{\perp}-$ пространство, ортогональное к $U$ в $F_{2}^{n}$. Пусть $v$ - произвольный вектор из $F_{2}^{n}$. Тогда

$$
\sum_{u \in U+v} W_{f}(u)=2^{l} \sum_{x \in U^{\perp}}(-1)^{f(x)+\langle x, v\rangle} .
$$

Лемма 7. Пусть $f-$ платовидная функиия с носителем спектра мощности $4^{h}$, заданная на $F_{2}^{n}$. Тогда

$$
\sum_{a \in F_{2}^{n}} \varphi(a) \in\left\{-2^{h}, 2^{h}\right\} .
$$

Доказательство. Возьмем в лемме 6 в качестве подпространства $U$ все $F_{2}^{n}$. В обозначениях леммы 6

$$
W_{f}(u)=\varphi(u) 2^{n-h}, \quad l=n .
$$

Тогда $U^{\perp}=\{0\}$. Поэтому

$$
\sum_{u \in F_{2}^{n}} W_{f}(u)=2^{n}(-1)^{f(0)}
$$

Отсюда следует, что

$$
\sum_{u \in F_{2}^{n}} \varphi(u)=2^{h}(-1)^{f(0)} \in\left\{-2^{h}, 2^{h}\right\}
$$

Лемма 8. Пусть $f$ - платовидная функция с носителем спектра мошности $4^{h}$, заданная на $F_{2}^{n}$. Пусть

$$
\sum_{a \in F_{2}^{n}} \varphi(a)=2^{h}
$$


Пусть $Н$ является $(n-1)$-мерным классом смежности в $F_{2}^{n}$. Тогда

$$
\sum_{a \in H} \varphi(a) \in\left\{0,2^{h}\right\}
$$

Доказательство. В обозначениях леммы 6

$$
W_{f}(u)=\varphi(u) 2^{n-h}, \quad l=n-1 .
$$

Поэтому из леммы 6 следует, что

$$
\sum_{a \in H} \varphi(a) \in\left\{-2^{h}, 0,2^{h}\right\}
$$

Но если сумма равна $-2^{h}$, то $\sum_{a \in F_{2}^{n} \backslash H} \varphi(a)=2^{h+1}$, что невозможно по сказанному выше.

Следующее утверждение также хорошо известно (см., например, теорему 2.89 в [2]).

Лемма 9. Пусть $f$ - булева функиия на $F_{2}^{n}$. Пусть $U$ является линейным подпространством в $F_{2}^{n}$ размерности $l, a U^{\perp}$ - пространство, ортогональное $\kappa U$ в $F_{2}^{n}$. Тогда

$$
\sum_{u \in U} W_{f}^{2}(u)=2^{l} \sum_{v \in U^{\perp}} \Delta_{f}(v)
$$

Лемма 10. Пусть $f-$ булева функчия на $F_{2}^{n}, n \geqslant 1$. Тогда wt $(f)$ нечетно тогда и только тогда, когда $\operatorname{deg}(f)=n$.

Доказательство очевидно (см., например, следствие 1 в [3]).

Лемма 11. Пусть $f$ - булева функчия на $F_{2}^{n}$, не равная тождественно постоянной. Тогда

$$
2^{n-\operatorname{deg}(f)} \leqslant \operatorname{wt}(f) \leqslant 2^{n}-2^{n-\operatorname{deg}(f)} .
$$

Доказательство очевидно (см., например, лемму 5.6 в [2] или лемму 3 в [3]).

Лемма 12 ([9]). Пусть $f-$ платовидная булева функиия на $F_{2}^{n}$ с носителем спектра мочности $4^{h}$. Тогда $\operatorname{deg}(f) \leqslant h+1$.

Доказательство. Коэффициенты Уолша функции $f$ принимают значения из множества $\left\{0, \pm 2^{n-h}\right\}$. Рассмотрим самое длинное слагаемое $x_{i_{1}} x_{i_{2}} \ldots x_{i_{s}}$ функции $f$ (если таких слагаемых несколько, берем любое из них). Можно считать, что $s \geqslant 2$, иначе утверждение автоматически является верным. Воспользуемся леммой 6. Возьмем в качестве $U$ линейное подпространство $U=\left\{x \in F_{2}^{n} \mid x_{i_{1}}=0, \ldots, x_{i_{s}}=0\right\}$ размерности $l=n-s$, вектор $v$ возьмем нулевым. Тогда ортогональное пространство $U^{\perp}$ по лемме 10 содержит нечетное число наборов $x$ таких, что $f(x)=1$. Поэтому сумма $\sum_{x \in U^{\perp}}(-1)^{f(x)}$ при $s \geqslant 2$ не делится на 4. Следовательно, в равенстве

$$
\sum_{u \in U} W_{f}(u)=2^{n-s} \sum_{x \in U^{\perp}}(-1)^{f(x)}
$$

левая часть делится на $2^{n-h}$, а правая часть не делится на $2^{n-s+2}$. Отсюда следует, что $n-h<n-s+2$, и учитывая целочисленность, получаем, что $s \leqslant h+1$, что и требовалось доказать. 
Лемма 13. Пусть $f$ - платовидная булева функция на $F_{2}^{n}$ с носителем спектра мощности $4^{h}$. Пусть $Н$ является $(n-1)$-мерным классом смежности в $F_{2}^{n}$. Тогда либо $\sum_{u \in H}|\varphi(u)|=0$, либо $\sum_{u \in H}|\varphi(u)|=4^{h}$, либо $2^{h} \leqslant \sum_{u \in H}|\varphi(u)| \leqslant 4^{h}-2^{h}$.

Доказательство. Пусть $H-$ линейное подпространство в $F_{2}^{n}$. Тогда $H^{\perp}=\{0, v\}$ для некоторого ненулевого $v \in F_{2}^{n}$. Очевидно, $\Delta_{f}(0)=2^{n}$. По лемме 9

$$
4^{n-h} \sum_{u \in H}|\varphi(u)|=2^{n-1}\left(2^{n}+\Delta_{f}(v)\right)=4^{n}-2^{n} \operatorname{wt}\left(D_{v} f\right)
$$

В силу леммы 12 справедливо неравенство $\operatorname{deg}(f) \leqslant h+1$. Функция $D_{v} f$ является производной функции $f$, поэтому $\operatorname{deg}\left(D_{v} f\right) \leqslant h$. Если $D_{v} f \equiv 0$, то $\sum_{u \in H}|\varphi(u)|=4^{h}$. Если $D_{v} f \equiv 1$, то $\sum_{u \in H}|\varphi(u)|=0$. Если $D_{v} f$ не является постоянной, то в силу леммы 11

$$
2^{n-h} \leqslant \operatorname{wt}\left(D_{v} f\right) \leqslant 2^{n}-2^{n-h} .
$$

Отсюда, $2^{h} \leqslant \sum_{u \in H}|\varphi(u)| \leqslant 4^{h}-2^{h}$, что и требовалось доказать. Для класса смежности $F_{2}^{n} \backslash H$ точно такие же три случая имеют место в силу только что доказанного и равенства Парсеваля.

Лемма 14. Пусть $f_{1}, f_{2}$-булевы функчии на $F_{2}^{n}$, а $f$-булева функция на $F_{2}^{n+1}$, причем $f\left(x x_{n+1}\right)=\left(x_{n+1}+1\right) f_{1}(x)+x_{n+1} f_{2}(x)$. Тогда

$$
W_{f}(u 0)=W_{f_{1}}(u)+W_{f_{2}}(u), \quad W_{f}(u 1)=W_{f_{1}}(u)-W_{f_{2}}(u) .
$$

Эта лемма хорошо известна. Фактически на ее применении основано быстрое преобразование Уолша.

\section{4. Об аффинном ранге платовидных булевых функций с носителем спектра мощности 16}

Во всех утверждениях этого раздела предполагается, что $f$ - платовидная булева функция на $F_{2}^{n}$ и $\left|S_{f}\right|=16$. В этом случае $c=n-2$. По лемме 7 имеет место один из двух случаев $\left|T^{+}\right|=10,\left|T^{-}\right|=6$, или же $\left|T^{+}\right|=6,\left|T^{-}\right|=10$. Учитывая, что для всех $u$ справедливо равенство $W_{f}(u)=-W_{f+1}(u)$, можно без ограничения общности считать, что $\left|T^{+}\right|=10$, $\left|T^{-}\right|=6$, что мы и будем делать в дальнейшем в этом разделе. Таким образом, $\left|S_{f}\right|=16$, $\left|T^{+}\right|=10,\left|T^{-}\right|=6$.

Нашей целью является доказательство следующей теоремы.

Теорема 1. Пусть $f$ является платовидной функиией, $\left|S_{f}\right|=16$. Тогда для аффинного ранга $k_{a}$ носителя спектра $S_{f}$ справедливо неравенство $k_{a} \leqslant 6$.

Доказательство теоремы 1 будет получено путем доказательства серии лемм.

Предположим, что аффинный ранг носителя спектра $S_{f}$ равен $k_{a}$ и аффинный ранг $T^{-}$равен $k^{-}$. Очевидно, $3 \leqslant k^{-} \leqslant 5$. Легко видеть, что с помощью некоторого аффинного отображения в $F_{2}^{n}$ можно вложить наименьший класс смежности, содержащий носитель спектра $S_{f}$, в $F_{2}^{k_{a}} \otimes(0, \ldots, 0)$, так чтобы некоторые $k^{-}+1$ наборов из $T^{-}$перешли в наборы $(0,0,0, \ldots, 0),(1,0,0, \ldots, 0),(0,1,0, \ldots, 0), \ldots,(0,0, \ldots, 0,1,0, \ldots, 0)$, где в последнем наборе 1 стоит на месте $k^{-}$. Заметим, что после такого отображения все наборы 
из $T^{-}$перейдут в наборы, имеющие только нули во всех компонентах $i, i>k^{-}$. Отметим, что описанное выше аффинное отображение спектра не является, вообще говоря, аффинным преобразованием функции $f$, однако нам этого и не нужно. Нам достаточно того, что получившаяся в результате отображения булева функция будет платовидной с тем же наборов значений, принимаемых коэффициентами Уолша, и теми же значениями $k_{a}$ и $k^{-}$. По лемме 3 переменные с $\left(k_{a}+1\right)$-й по $n$-ю у получившейся функции будут фиктивными. Отбрасывая их и деля все коэффициенты Уолша на $2^{n-k_{a}}$, мы по леммам 2 и 3 получим платовидную функцию, заданную на $F_{2}^{k_{a}}$, с носителем спектра той же мощности 16. Таким образом, без потери общности в оставшейся части этого раздела мы будем рассматривать именно такой носитель спектра.

Лемма 15. Пусть $H$ является $\left(k_{a}-1\right)$-мерным классом смежности в $F_{2}^{k_{a}}$. Тогда

$$
\sum_{a \in H} \varphi(a) \in\{0,4\}
$$

Утверждение леммы является частным случаем леммы 8.

Лемма 16 ([5]). Пусть $Н$ является $\left(k_{a}-1\right)$-мерным классом смежности в $F_{2}^{k_{a}}$. Тогда $H$ содержит 4, 6, 8, 10 или 12 наборов из $S_{f}$.

Доказательство. В силу леммы 15 класс $H$ содержит четное число наборов из $S_{f}$. Случаи 2 и 14 невозможны в силу леммы 13. Если $H$ содержит 16 наборов из $S_{f}$, то $S_{f}$ содержится в $H$; если $H$ содержит 0 наборов из $S_{f}$, то $S_{f}$ содержится в $F_{2}^{k_{a}} \backslash H$. Оба последних случая невозможны в силу того, что $F_{2}^{k_{a}}$ - наименьший класс смежности, содержащий носитель спектра $S_{f}$.

Наша цель - доказать, что $k_{a} \leqslant 6$. Предположим противное. Пусть $k_{a} \geqslant 7$. Докажем, что это невозможно.

Образуем матрицу $M$ размера $16 \times 7$. В строках $M$ будем записывать слева направо первые 7 компонент наборов из $S_{f}$ (в случае $k_{a}>7$ мы опустим все компоненты после седьмой). В первых 10 строках $M$ запишем наборы из $T^{+}$, а в последних 6 строках $M$ запишем наборы из $T^{-}$. Левые $k^{-}$столбцов $M$ назовем левой частью $M$, оставшиеся $7-k^{-}$столбцов назовем правой частью $M$.

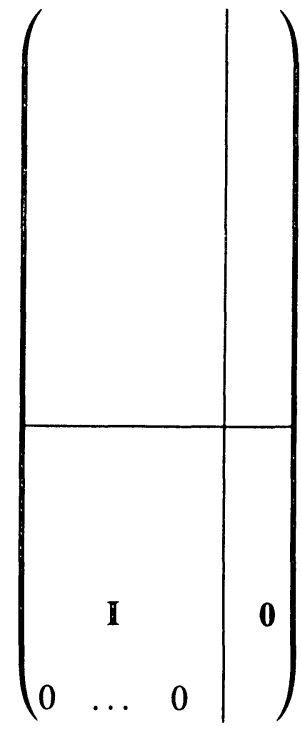


Обозначим через $\gamma_{i}$ столбцы из левой части $M$, а через $x_{i}$ соответствующие этим столбцам переменные. Обозначим через $\delta_{j}$ столбцы из правой части $M$, а через $y_{j}$ соответствующие этим столбцам переменные. Обозначим через $\gamma_{i}^{+}$и $\delta_{j}^{+}$подстолбцы, содержащие верхние 10 элементов столбцов $\gamma_{i}$ и $\delta_{j}$, соответственно.

Лемма 17. Для любого множества $\delta_{j_{1}}, \ldots, \delta_{j_{s}}, 1 \leqslant s \leqslant 7-k^{-}$, различных столбчов из правой части М справедливо равенство $\operatorname{wt}\left(\delta_{j_{1}}^{+}+\ldots+\delta_{j_{s}}^{+}\right)=4$.

Доказательство. Положим $H=\left\{F_{2}^{k_{a}} \mid y_{j_{1}}+\ldots+y_{j_{s}}=0\right\}$. Гиперплоскость $H$ содержит все 6 наборов из $T^{-}$, поэтому по лемме 15 гиперплоскость $H$ должна содержать 6 или 10 наборов из $T^{+}$. Но если $H$ содержит 10 наборов из $T^{+}$, то $H$ содержит $S_{f}$. Это невозможно, поскольку $k_{a}$ есть размерность наименьшего класса смежности, содержащего $S_{f}$. Поэтому $H$ содержит 12 наборов из $S_{f}$ и $F_{2}^{n} \backslash H$ содержит в точности 4 набора из $S_{f}$.

Лемма 18. Существует не более трех столбцов, удовлетворяющих условию леммы 17. Без потери общности можно выбрать в качестве этих столбчов

$$
\begin{aligned}
& \delta_{1}^{+}=(0,0,0,0,0,0,1,1,1,1)^{T}, \\
& \delta_{2}^{+}=(0,0,0,0,1,1,0,0,1,1)^{T}, \\
& \delta_{3}^{+}=(0,0,0,1,0,1,0,1,0,1)^{T}
\end{aligned}
$$

Доказательство. Легко проверить, что наборы $\delta_{1}^{+}, \delta_{2}^{+}, \delta_{3}^{+}$, указанные в условии, могут быть взяты без потери общности. Предположим, что можно добавить к этому множеству некоторый набор $\delta_{4}^{+}$. Для $c_{1}, c_{2}, c_{3} \in\{0,1\}$ положим

$$
\delta^{+}\left(c_{1}, c_{2}, c_{3}\right)=c_{1} \delta_{1}^{+}+c_{2} \delta_{2}^{+}+c_{3} \delta_{3}^{+} .
$$

Рассмотрим сумму

$$
S=\sum_{c_{1}, c_{2}, c_{3} \in\{0,1\}} d\left(\delta_{4}^{+}, \delta^{+}\left(c_{1}, c_{2}, c_{3}\right)\right) .
$$

Заметим, что для любой строки с 4-й по 10-ю в точности 4 из 8 наборов $\delta^{+}\left(c_{1}, c_{2}, c_{3}\right)$ имеют единицу в этой строке. Поэтому $S=28+8 w_{0}=32$, где $w_{0}$ - это число единиц в $\delta_{4}^{+}$в строках с 1-й по 3-ю. Отсюда следует, что $w_{0}=0,5$, но $w_{0}$ должно быть целым числом. Это противоречие доказывает лемму 18.

Лемма 19. Правая часть матричы $M$ содержит не более 3 столбчов.

Утверждение леммы следует из лемм 17 и 18.

Из леммы 19 следует, что случай $k^{-}=3$ невозможен. Остались случаи $k^{-}=4$ и $k^{-}=5$.

Лемма 20. Пусть $\gamma_{i}-$ столбеи из левой части матрицы $M$. Предположим, что $\gamma_{i}$ содержит одну единичу и 5 нулей в нижних 6 строках. Тогда $\operatorname{wt}\left(\gamma_{i}^{+}\right)=5$.

Доказательство. Положим $H=\left\{F_{2}^{k_{a}} \mid x_{i}=0\right\}$. Гиперплоскость $H$ содержит в точности 5 наборов из $T^{-}$, поэтому по лемме 15 гиперплоскость $H$ должна содержать 5 или 9 наборов из $T^{+}$. Но если $H$ содержит 9 наборов из $T^{+}$, то $H$ содержит в точности 14 наборов из $S_{f}$. Это невозможно по лемме 16 . Отсюда следует, что $\operatorname{wt}\left(\gamma_{i}^{+}\right)=5$. 
Лемма 21. Пусть $\gamma_{i}$ - столбеч из левой части матрищы $M$. Предположим, что $\gamma_{i}$ содержит 2 единицы и 4 нуля в нижних 6 строках. Тогда $\operatorname{wt}\left(\gamma_{i}^{+}\right) \in\{2,6\}$.

Доказательство. Положим $H=\left\{F_{2}^{k_{a}} \mid x_{i}=0\right\}$. Гиперплоскость $H$ содержит в точности 4 набора из $T^{-}$, поэтому по лемме 15 гиперплоскость $H$ должна содержать 4 или 8 наборов из $T^{+}$. Отсюда следует, что $\operatorname{wt}\left(\gamma_{i}^{+}\right)$равно 2 или 6.

Рассмотрим теперь отдельно случаи $k^{-}=4$ и $k^{-}=5$.

Случай $k^{-}=5$. В этом случае правая часть матрицы $M$ содержит два столбца. По лемме 18 без ограничения общности можно предположить, что эти столбцы суть

$$
\delta_{1}=(0,0,0,0,0,0,1,1,1,1)^{T}, \quad \delta_{2}=(0,0,0,0,1,1,0,0,1,1)^{T} .
$$

Без ограничения общности можно считать, что матрица $M$ имеет вид

$$
\left(\begin{array}{lllll|ll}
* & * & * & * & * & 0 & 0 \\
* & * & * & * & * & 0 & 0 \\
* & * & * & * & * & 0 & 0 \\
* & * & * & * & * & 0 & 0 \\
* & * & * & * & * & 0 & 1 \\
* & * & * & * & * & 0 & 1 \\
* & * & * & * & * & 1 & 0 \\
* & * & * & * & * & 1 & 0 \\
* & * & * & * & * & 1 & 1 \\
* & * & * & * & * & 1 & 1 \\
\hline 1 & 0 & 0 & 0 & 0 & 0 & 0 \\
0 & 1 & 0 & 0 & 0 & 0 & 0 \\
0 & 0 & 1 & 0 & 0 & 0 & 0 \\
0 & 0 & 0 & 1 & 0 & 0 & 0 \\
0 & 0 & 0 & 0 & 1 & 0 & 0 \\
0 & 0 & 0 & 0 & 0 & 0 & 0
\end{array}\right) .
$$

По лемме 20 все столбцы $\gamma_{i}^{+}, i=1,2,3,4,5$, содержат в точности 5 единиц.

Лемма 22. Пусть $k^{-}=5$. Тогда при $1 \leqslant i_{1}<i_{2} \leqslant 5$ справедливо включение $d\left(\gamma_{i_{1}}^{+}, \gamma_{i_{2}}^{+}\right) \in\{2,6\}$.

Доказательство. Положим $H=\left\{F_{2}^{k_{a}} \mid x_{i_{1}}+x_{i_{2}}=0\right\}$. Гиперплоскость $H$ содержит в точности 4 набора из $T^{-}$, поэтому по лемме 15 гиперплоскость $H$ должна содержать 4 или 8 наборов из $T^{+}$. Отсюда следует, что

$$
d\left(\gamma_{i_{1}}^{+}, \gamma_{i_{2}}^{+}\right)=\operatorname{wt}\left(\gamma_{i_{1}}^{+}+\gamma_{i_{2}}^{+}\right) \in\{2,6\} .
$$

Лемма 23. Пусть $k^{-}=5$. Тогда для любых $i \in\{1,2,3,4,5\}$ u $c_{1}, c_{2} \in\{0,1\}$

$$
d\left(\gamma_{i}^{+}, c_{1} \delta_{1}^{+}+c_{2} \delta_{2}^{+}\right)=5 \text {. }
$$

Доказательство. Положим $H=\left\{F_{2}^{k_{a}} \mid x_{i}+c_{1} y_{1}+c_{2} y_{2}=0\right\}$. Гиперплоскость $H$ содержит в точности 5 наборов из $T^{-}$, поэтому по лемме 15 гиперплоскость $H$ должна содержать 5 или 9 наборов из $T^{+}$. Но если $H$ содержит 9 наборов из $T^{+}$, то $H$ содержит в точности 14 наборов из $S_{f}$. Это невозможно по лемме 16 . Отсюда следует, что

$$
d\left(\gamma_{i}^{+}, c_{1} \delta_{1}^{+}+c_{2} \delta_{2}^{+}\right)=\operatorname{wt}\left(\gamma_{i}^{+}+c_{1} \delta_{1}^{+}+c_{2} \delta_{2}^{+}\right)=5 \text {. }
$$


Лемма 24. Пусть $k^{-}=5$. Тогда для любого $i, i \in\{1,2,3,4,5\}$, столбеч $\gamma_{i}^{+}$содержит в точности 2 единичы в строках с 1-й по 4-ю, в точности 1 единичу в строках 5, 6, в точности 1 единичу в строках 7, 8, в точности 1 единичу в строках 9, 10.

Доказательство. Если $\gamma_{i}^{+}$содержит 0 единиц в строках 9, 10, то из равенства $d\left(\gamma_{i}^{+}, \delta_{1}^{+}\right)=d\left(\gamma_{i}^{+}, \delta_{2}^{+}\right)=5$ следует, что $\gamma_{i}^{+}$содержит только единицы в строках 5,6 , 7 , 8. Но в этом случае $d\left(\gamma_{i}^{+}, \delta_{1}^{+}+\delta_{2}^{+}\right)=1$, что противоречит лемме 23 . Если $\gamma_{i}^{+}$содержит 2 единицы в строках 9,10 , то из равенства $d\left(\gamma_{i}^{+}, \delta_{1}^{+}\right)=d\left(\gamma_{i}^{+}, \delta_{2}^{+}\right)=5$ следует, что $\gamma_{i}^{+}$содержит только нули в строках $5,6,7,8$. Но в этом случае $d\left(\gamma_{i}^{+}, \delta_{1}^{+}+\delta_{2}^{+}\right)=9$, что противоречит лемме 23. Поэтому $\gamma_{i}^{+}$содержит в точности 1 единицу в строках 9 , 10. Отсюда следует, что $\gamma_{i}^{+}$содержит в точности 1 единицу в строках 7,8 , в точности 1 единищу в строках 5, 6 и в точности 2 единицы в строках с 1-й по 4-ю.

Лемма 25. Случай $k^{-}=5$ невозможен.

Доказательство. Существует ровно 3 пары противоположных наборов длины 4 с в точности 2 единицами. Поэтому в левой части $M$ найдутся два столбца $\gamma_{i_{1}}$ и $\gamma_{i_{2}}$, которые либо идентичны, либо противоположны в верхних 4 строках. Пусть $\gamma_{i_{3}}$ - еще какой-то столбец в левой части $M, i_{1} \neq i_{3}, i_{2} \neq i_{3}$. Тогда из леммы 24 легко видеть, что каждая группа строк $(1-4),(5,6),(7,8),(9,10)$ дает в сумму $S=d\left(\gamma_{i_{1}}^{+}, \gamma_{i_{2}}^{+}\right)+d\left(\gamma_{i_{1}}^{+}, \gamma_{i_{3}}^{+}\right)+d\left(\gamma_{i_{2}}^{+}, \gamma_{i_{3}}^{+}\right)$ вклад, делящийся на 4 . Поэтому сумма $S$ делится на 4. С другой стороны, по лемме 22 все слагаемые в $S$ сравнимы с 2 по модулю 4 . Поэтому $S$ также сравнима с 2 по модулю 4. Это противоречие доказывает лемму 25.

Таким образом, мы доказали, что случай $k^{-}=5$ невозможен.

Случай $k^{-}=4$. В этом случае правая часть матрицы $M$ содержит в точности три столбца. По лемме 18 без ограничения общности мы можем предположить, что эти столбцы есть

$$
\begin{aligned}
& \delta_{1}=(0,0,0,0,0,0,1,1,1,1)^{T}, \\
& \delta_{2}=(0,0,0,0,1,1,0,0,1,1)^{T}, \\
& \delta_{3}=(0,0,0,1,0,1,0,1,0,1)^{T} .
\end{aligned}
$$

Без ограничения общности мы можем предположить, что матрица $M$ имеет вид

$$
\left(\begin{array}{cccc|ccc}
* & * & * & * & 0 & 0 & 0 \\
* & * & * & * & 0 & 0 & 0 \\
* & * & * & * & 0 & 0 & 0 \\
* & * & * & * & 0 & 0 & 1 \\
* & * & * & * & 0 & 1 & 0 \\
* & * & * & * & 0 & 1 & 1 \\
* & * & * & * & 1 & 0 & 0 \\
* & * & * & * & 1 & 0 & 1 \\
* & * & * & * & 1 & 1 & 0 \\
* & * & * & * & 1 & 1 & 1 \\
\hline * & * & * & * & 0 & 0 & 0 \\
1 & 0 & 0 & 0 & 0 & 0 & 0 \\
0 & 1 & 0 & 0 & 0 & 0 & 0 \\
0 & 0 & 1 & 0 & 0 & 0 & 0 \\
0 & 0 & 0 & 1 & 0 & 0 & 0 \\
0 & 0 & 0 & 0 & 0 & 0 & 0
\end{array}\right)
$$


Пусть $c_{1}, c_{2}, c_{3} \in\{0,1\}$. Положим

$$
\delta^{+}\left(c_{1}, c_{2}, c_{3}\right)=c_{1} \delta_{1}^{+}+c_{2} \delta_{2}^{+}+c_{3} \delta_{3}^{+} .
$$

Лемма 26. Пусть $k^{-}=4$. Тогда никакой столбеч $\gamma_{i}$ в левой части матричы $М$ не может иметь ноль в 11-й строке.

Доказательство. Предположим, что некоторый столбец $\gamma_{i}$ имеет ноль в 11-й строке. Тогда по лемме 20 имеет место равенство $\mathrm{wt}\left(\gamma_{i}^{+}\right)=5$. Тем же путем, что и в доказательстве леммы 23, можно показать, что для любых $c_{1}, c_{2}, c_{3} \in\{0,1\}$ справедливо равенство $d\left(\gamma_{i}^{+}, \delta^{+}\left(c_{1}, c_{2}, c_{3}\right)\right)=5$. Рассмотрим сумму

$$
S=\sum_{c_{1}, c_{2}, c_{3} \in\{0,1\}} d\left(\gamma_{i}^{+}, \delta^{+}\left(c_{1}, c_{2}, c_{3}\right)\right) .
$$

Заметим, что для любой строки с 4-й по 10-ю в точности 4 из 8 наборов $\delta^{+}\left(c_{1}, c_{2}, c_{3}\right)$ имеют единицу в этой строке. Поэтому $S=28+8 w_{0}=40$, где $w_{0}$ - это число единиц в $\gamma_{i}$ в строках с 1-й по 3-ю. Отсюда следует, что $w_{0}=1,5$, но $w_{0}$ должно быть целым числом. Это противоречие доказывает лемму 26.

Из леммы 26 следует, что без ограничения общности матрица $M$ имеет вид

$$
\left(\begin{array}{llll|lll}
* & * & * & * & 0 & 0 & 0 \\
* & * & * & * & 0 & 0 & 0 \\
* & * & * & * & 0 & 0 & 0 \\
* & * & * & * & 0 & 0 & 1 \\
* & * & * & * & 0 & 1 & 0 \\
* & * & * & * & 0 & 1 & 1 \\
* & * & * & * & 1 & 0 & 0 \\
* & * & * & * & 1 & 0 & 1 \\
* & * & * & * & 1 & 1 & 0 \\
* & * & * & * & 1 & 1 & 1 \\
\hline 1 & 1 & 1 & 1 & 0 & 0 & 0 \\
1 & 0 & 0 & 0 & 0 & 0 & 0 \\
0 & 1 & 0 & 0 & 0 & 0 & 0 \\
0 & 0 & 1 & 0 & 0 & 0 & 0 \\
0 & 0 & 0 & 1 & 0 & 0 & 0 \\
0 & 0 & 0 & 0 & 0 & 0 & 0
\end{array}\right)
$$

Лемма 27. Пусть $k^{-}=4$. Тогда $d\left(\gamma_{i_{1}}^{+}, \gamma_{i_{2}}^{+}\right) \in\{2,6\}$ для любых $1 \leqslant i_{1}<i_{2} \leqslant 4$.

Доказательство. Положим $H=\left\{F_{2}^{k_{a}} \mid x_{i_{1}}+x_{i_{2}}=0\right\}$. Гиперплоскость $H$ содержит в точности 4 набора из $T^{-}$, поэтому по лемме 15 гиперплоскость $H$ должна содержать 4 или 8 наборов из $T^{+}$. Отсюда следует, что $d\left(\gamma_{i_{1}}^{+}, \gamma_{i_{2}}^{+}\right) \in\{2,6\}$.

Лемма 28. Пусть $k^{-}=4$. Тогда любой столбеч $\gamma_{i}$ в левой части матрицы $M$ имеет в точности 2 единицы в строках с 1-й по 3-ю и совпадает в строках с 4-й по 10-ю $c$ вектор-столбиом $\delta^{+}\left(c_{1}, c_{2}, c_{3}\right)$ при некоторых $c_{1}, c_{2}, c_{3} \in\{0,1\}$. 
Доказательство. В силу леммы 21 справедливо включение $\operatorname{wt}\left(\gamma_{i}^{+}\right) \in\{2,6\}$. Тем же способом можно показать, что для любых $c_{1}, c_{2}, c_{3} \in\{0,1\}$ справедливо включение $d\left(\gamma_{i}^{+}, \delta^{+}\left(c_{1}, c_{2}, c_{3}\right)\right) \in\{2,6\}$.

Предположим, что $\mathrm{wt}\left(\gamma_{i}^{+}\right)=2$. Если $\gamma_{i}^{+}$не содержит обе свои единицы в строках с 1-й по 3-ю, то легко найти $c_{1}, c_{2}, c_{3} \in\{0,1\}$ такие, что набор $\delta^{+}\left(c_{1}, c_{2}, c_{3}\right)$ содержит в точности одну единищу в тех строках, где и $\gamma_{i}^{+}$имеет единицу. Тогда будет справедливо равенство $d\left(\gamma_{i}^{+}, \delta^{+}\left(c_{1}, c_{2}, c_{3}\right)\right)=4$, что невозможно. Поэтому $\gamma_{i}^{+}$содержит обе свои единицы в строках с 1-й по 3-ю и совпадает в строках с 4-й по 10-ю с набором $\delta^{+}(0,0,0)$, то есть имеет требуемый вид.

Теперь предположим, что $\mathrm{wt}\left(\gamma_{i}^{+}\right)=6$. Рассмотрим сумму

$$
S=\sum_{c_{1}, c_{2}, c_{3} \in\{0,1\}} d\left(\gamma_{i}^{+}, \delta^{+}\left(c_{1}, c_{2}, c_{3}\right)\right) \text {. }
$$

Заметим, что для любой строки с 4-й по 10-ю в точности 4 из 8 наборов $\delta^{+}\left(c_{1}, c_{2}, c_{3}\right)$ имеют единицу в этой строке. Поэтому $S=28+8 w_{0}$, где $w_{0}-$ число единиц в $\gamma_{i}$ в строках с 1-й по 3-ю. Если для любых $c_{1}, c_{2}, c_{3} \in\{0,1\}$ справедливо равенство $d\left(\gamma_{i}^{+}, \delta^{+}\left(c_{1}, c_{2}, c_{3}\right)\right)=6$, то $S=48$ и $w_{0}=2,5$. Но $w_{0}$ должно быть целым числом. Поэтому существуют $c_{1}, c_{2}, c_{3} \in\{0,1\}$ такие, что $d\left(\gamma_{i}^{+}, \delta^{+}\left(c_{1}, c_{2}, c_{3}\right)\right)=2$. Обозначим $\delta^{+}\left(c_{1}, c_{2}, c_{3}\right)$ через $\delta_{0}^{+}$. Тогда wt $\left(\gamma_{i}^{+}+\delta_{0}^{+}\right)=2$ и для любых $c_{1}, c_{2}, c_{3} \in\{0,1\}$ справедливо включение $d\left(\gamma_{i}^{+}+\delta_{0}^{+}, \delta^{+}\left(c_{1}, c_{2}, c_{3}\right)\right) \in\{2,6\}$. Как было указано в начале этого доказательства, набор $\gamma_{i}^{+}+\delta_{0}^{+}$должен иметь в точности 2 единицы в строках с 1-й по 3-ю и одни нули в строках с 4-й по 10-ю. Следовательно, набор $\gamma_{i}^{+}$имеет в точности 2 единиц в строках с 1-й по 3 и совпадает с набором $\delta_{0}^{+}$в строках с 4-й по 10-ю.

Лемма 29. Случай $k^{-}=4$ невозможен.

Доказательство. По лемме 28 все столбцы $\gamma_{i}$ в левой части $M$ имеют в точности 2 единицы в строках с 1-й по 3-ю. Левая часть $M$ содержит 4 столбца, поэтому среди них найдутся столбцы $\gamma_{i_{1}}$ и $\gamma_{i_{2}}, 1 \leqslant i_{1}<i_{2} \leqslant 4$, совпадающие в строках с 1-й по 3-ю. В строках с 4-й по 10-ю столбцы $\gamma_{i_{1}}$ и $\gamma_{i_{2}}$ совпадают по лемме 28 с некоторыми вектор-столбцами $\delta^{+}\left(c_{1}^{\prime}, c_{2}^{\prime}, c_{3}^{\prime}\right)$ и $\delta^{+}\left(c_{1}^{\prime \prime}, c_{2}^{\prime \prime}, c_{3}^{\prime \prime}\right)$, соответственно. В силу леммы 17 справедливо включение $d\left(\delta^{+}\left(c_{1}^{\prime}, c_{2}^{\prime}, c_{3}^{\prime}\right), \delta^{+}\left(c_{1}^{\prime \prime}, c_{2}^{\prime \prime}, c_{3}^{\prime \prime}\right)\right) \in\{0,4\}$. Отсюда, $d\left(\gamma_{i_{1}}^{+}, \gamma_{i_{2}}^{+}\right) \in\{0,4\}$, что противоречит лемме 27.

Все случаи рассмотрены. Теорема 1 доказана.

Таким образом, мы показали, что аффинный ранг платовидных функций с носителем спектра мощности 16 не может принимать никаких значений, кроме 4, 5 и 6. Функции с такими параметрами известны, и их примеры приводились, например, в [5]. Мы не будем здесь приводить примеры. Эти примеры построены в следующем разделе в рамках общей конструкщии.

\section{5. Оценки аффинного ранга платовидных функций с произвольной мощностью носителя спектра}

Лемма 30. Пусть существует платовидная функиия с носителем спектра мощности $4^{h}$ и аффинньм рангом $k_{a}$. Тогда для любого натурального $s$, удовлетворяющего неравенствам $k_{a}+2 \leqslant s \leqslant 2 k_{a}+2$, существует платовидная функция с носителем спектра мочности $4^{h+1}$ и аффинньм рангом $s$. 
Доказательство. Если существует платовидная функщия с носителем спектра мощности $4^{h}$ и аффинным рангом $k_{a}$, то из нее по леммам 2 и 3 аффинным преобразованием спектра и последующим удалением фиктивных переменных можно получить платовидную функцию $f$ на $F_{2}^{k_{a}}$ с носителем спектра мощности $4^{h}$, причем так, чтобы спектру функции $f$ принадлежал и нулевой набор, и все наборы веса 1. Рассмотрим функцию $f_{1}\left(x_{1}, \ldots, x_{s}\right)=f\left(x_{s-k_{a}}, \ldots, x_{s-1}\right)+x_{s}$ на $F_{2}^{s}$ (переменные $x_{1}, \ldots, x_{s-k_{a}-1}$ у функции $f_{1}$ будут фиктивными). По леммам 3 и 4 функция $f_{1}$ снова будет платовидной с той же мощностью носителя, причем ко всем наборам из $S_{f}$ в носителе спектра $S_{f_{1}}$ слева припишется $s-k_{a}-1$ нулей, а справа припишется единица. Линейное подпространство размерности $k_{a}$ в $F_{2}^{k_{a}}$, содержавшее $S_{f}$, при переходе к функции $f_{1}$ перейдет в класс смежности размерности $k_{a}$ в $F_{2}^{s}$, содержащий $S_{f_{1}}$, но линейным подпространством не являющийся. Поэтому ранг функции $f_{1}$ равен $k_{a}+1$. Заметим, что носителю спектра $S_{f_{1}}$ принадлежат следующие наборы: все наборы веса 2 с единицами в компонентах $i$ и $s$, $i=s-k_{a}, \ldots, s-1$, а также набор веса 1 с единицей в компоненте $s$. Образуем функцию $f_{2}\left(x_{1}, \ldots, x_{s}\right)=f_{1}\left(x_{s}, \ldots, x_{1}\right)$ на $F_{2}^{s}$, переименовав все переменные в обратном порядке. Ясно, что функщия $f_{2}$ будет обладать свойствами, аналогичными свойствам функции $f_{1}$. Носителю спектра $S_{f_{2}}$ принадлежат в числе прочих следующие наборы: все наборы веса 2 с единицами в компонентах 1 и $i, i=2, \ldots, k_{a}+1$, а также набор веса 1 с единицей в компоненте 1. Заметим, что во всех наборах из $S_{f_{1}}$ в первой компоненте ноль, а во всех наборах из $S_{f_{2}}$ в первой компоненте единица. Поэтому множества $S_{f_{1}}$ и $S_{f_{2}}$ в $F_{2}^{s}$ не пересекаются.

Рассмотрим функцию

$$
f^{\prime}\left(x_{1}, \ldots, x_{s+1}\right)=\left(x_{s+1}+1\right) f_{1}\left(x_{1}, \ldots, x_{s}\right)+x_{s+1} f_{2}\left(x_{1}, \ldots, x_{s}\right)
$$

на $F_{2}^{s+1}$. По лемме 14 для любого $u \in F_{2}^{s}$

$$
W_{f^{\prime}}(u 0)=W_{f_{1}}(u)+W_{f_{2}}(u), \quad W_{f}(u 1)=W_{f_{1}}(u)-W_{f_{2}}(u) .
$$

Как было указано выше, множества $S_{f_{1}}$ и $S_{f_{2}}$ в $F_{2}^{s}$ не пересекаются. Поэтому каждый набор $u$ из $S_{f_{1}}$ и $S_{f_{2}}$ в $F_{2}^{s}$ даст ровно два набора $(u, 0)$ и $(u, 1)$, входящие в носитель спектра $S_{f^{\prime}}$ функции $f^{\prime}$ на $F_{2}^{s+1}$, причем значения ненулевых коэффициентов Уолша функщии $f^{\prime}$ будут теми же самыми, что и значения ненулевых коэффициентов Уолша функций $f_{1}$ и $f_{2}$. Таким образом, мощность $S_{f^{\prime}}$ равна $4^{h+1}$, и функция $f^{\prime}$ также является платовидной функцией.

Из сказанного выше следует, что $S_{f^{\prime}}$ принадлежат все наборы веса 2 с единицами в компонентах 1 и $i, i=2, \ldots, k_{a}+1$, все наборы веса 2 с единицами в компонентах $i$ и $s, i=s-k_{a}, \ldots, s-2, s-1, s+1$, а также наборы веса 1 с единицей в компонентах 1 и $s$. Легко видеть, что ранг вышеуказанной системы наборов равен $s+1$. Поэтому ранг функции $f^{\prime}$ на $F_{2}^{s+1}$ равен $s+1$. В то же время для любого набора из $S_{f^{\prime}}$ сумма значений 1-й и $s$-й компонент равна 1. Поэтому $S_{f}$ принадлежит гиперплоскости $H=\left\{x \in F_{2}^{s+1} \mid x_{1}+x_{s}=1\right\}$, и аффинный ранг функции $f^{\prime}$ меньше, чем $s+1$, но он не меньше, чем ранг функции $f^{\prime}$ без единицы, и поэтому аффинный ранг функции $f^{\prime}$ равен $s$. Таким образом, требуемая функция построена.

Теорема 2. Для любого натурального $k_{a}$, удовлетворяющего неравенствам $2 h \leqslant k_{a} \leqslant$ $2^{h+1}-2$, существует платовидная функция с носителем спектра мочности $4^{h}$ и аффинным рангом $k_{a}$. 
Доказательство. Проведем доказательство индукцией по $h$. При $h=1$ величина $k_{a}$ может быть равна только 2. Такой функцией является, например, бент-функция $x_{1} x_{2}$ на $F_{2}^{2}$ (если мы не хотим считать бент-функцию платовидной функцией, то добавим к ней фиктивную переменную). Если утверждение теоремы верно для $h$, то его справедливость для $h+1$ немедленно следует из леммы 30 .

Следствие 1. Аффинный ранг платовидной функции с носителем спектра мощности 16 может принимать только значения 4, 5 и 6.

Доказательство. Верхняя оценка $k_{a} \leqslant 6$ доказана в теореме 1 . Нижняя оценка $k_{a} \geqslant 4$ очевидна. Существование функщий с $k_{a}=4,5,6$ следует из теоремы 2. Заметим, что примеры таких функций даны в [5].

Тривиальной верхней оценкой аффинного ранга $k_{a}$ платовидной функции с носителем спектра мощности $4^{h}$ является $k_{a} \leqslant 4^{h}-1$. Приведем несколько улучшенную оценку.

Теорема 3. Пусть $f$ является платовидной функцией, $\left|S_{f}\right|=4^{h}$. Тогда для аффинного ранга $k_{a}$ носителя спектра $S_{f}$ справедлива оченка

$$
k_{a} \leqslant 2^{2 h-1}-2^{h-1}+h .
$$

Будем следовать путем, аналогичным доказательству теоремы 1. Согласно лемме 8 $\left|T^{+}\right|,\left|T^{-}\right| \in\left\{2^{2 h-1}+2^{h-1}, 2^{2 h-1}-2^{h-1}\right\}$. Без ограничения общности можно считать, что $\left|T^{+}\right|=2^{2 h-1}+2^{h-1},\left|T^{-}\right|=2^{2 h-1}-2^{h-1}$. Предположим, что аффинный ранг носителя спектра $S_{f}$ равен $k_{a}$ и аффинный ранг $T^{-}$равен $k^{-}$. Очевидно, что $k^{-} \leqslant 2^{2 h-1}-2^{h-1}-1$. Легко видеть, что с помощью некоторого аффинного отображения в $F_{2}^{n}$ можно вложить наименьший класс смежности, содержащий носитель спектра $S_{f}$, в $F_{2}^{k_{a}} \otimes(0, \ldots, 0)$, где $(0, \ldots, 0)-$ набор из $n-k_{a}$ нулей, так, чтобы некоторые $k^{-}+1$ наборов из $T^{-}$перешли в наборы $(0,0,0, \ldots, 0),(1,0,0, \ldots, 0),(0,1,0, \ldots, 0), \ldots,(0,0, \ldots, 0,1,0, \ldots, 0)$, где в последнем наборе единица стоит на $k^{-}$-м месте. Получившаяся в результате отображения булева функция будет платовидной с тем же набором значений, принимаемых коэффициентами Уолша, и теми же значениями $k_{a}$ и $k^{-}$. По лемме 3 переменные с $\left(k_{a}+1\right)$-й по $n$-ю у получившейся функции будут фиктивными. Отбрасывая их и деля все коэффициенты Уолша на $2^{n-k_{a}}$, мы по леммам 2 и 3 получим платовидную функщию, заданную на $F_{2}^{k_{a}}$, с носителем спектра той же мощности $2^{h}$. Таким образом, без потери общности мы в оставшейся части этого раздела будем рассматривать именно такой носитель спектра.

Образуем матрицу $M$ размера $4^{h} \times k_{a}$. В строках $M$ будем записывать слева направо наборы из $S_{f}$. В первых $2^{2 h-1}+2^{h-1}$ строках $M$ запишем наборы из $T^{+}$, а в последних $2^{2 h-1}-2^{h-1}$ строках $M$ запишем наборы из $T^{-}$. Левые $k^{-}$столбцов $M$ назовем левой частью $M$, оставшиеся $k_{a}-k^{-}$столбцов назовем правой частью $M$. Из того, что $\left|T^{-}\right|=2^{2 h-1}-2^{h-1}$, следует оценка $k^{-} \leqslant 2^{2 h-1}-2^{h-1}-1$.

Обозначим через $\delta_{j}$ столбцы из правой части $M$, а через $y_{j}$ соответствующие этим столбцам переменные. Обозначим через $\delta_{j}^{+}$подстолбцы, содержащие верхние $2^{2 h-1}+2^{h-1}$ элементов столбцов $\delta_{j}$ соответственно.

Лемма 31. Для любого множества $\delta_{j_{1}}, \ldots, \delta_{j_{s}}, 1 \leqslant s \leqslant k_{a}-k^{-}$, различных столбчов из правой части М выполняется равенство

$$
\operatorname{wt}\left(\delta_{j_{1}}^{+}+\ldots+\delta_{j_{s}}^{+}\right)=2^{h} .
$$


Доказательство. Положим $H=\left\{F_{2}^{k_{a}} \mid y_{j_{1}}+\ldots+y_{j_{s}}=0\right\}$. Гиперплоскость $H$ содержит все $2^{2 h-1}-2^{h-1}$ наборов из $T^{-}$, поэтому по лемме 8 гиперплоскость $H$ должна содержать $2^{2 h-1}-2^{h-1}$ или $2^{2 h-1}+2^{h-1}$ наборов из $T^{+}$. Но если $H$ содержит $2^{2 h-1}+2^{h-1}$ наборов из $T^{+}$, то $H$ содержит $S_{f}$. Это невозможно, поскольку $k_{a}$ есть размерность наименьшего класса смежности, содержащего $S_{f}$. Поэтому $H$ содержит $4^{h}-2^{h}$ наборов из $S_{f}$ и $F_{2}^{n} \backslash H$ содержит в точности $2^{h}$ наборов из $S_{f}$.

Лемма 32. Правая часть матрицы $M$ содержит не более $h+1$ столбчов.

Доказательство. Пусть правая часть матрицы $M$ содержит $m$ столбцов $\delta_{1}, \ldots, \delta_{m}$. Для $c_{1}, \ldots, c_{m} \in\{0,1\}$ положим $\delta^{+}\left(c_{1}, \ldots, c_{m}\right)=c_{1} \delta_{1}^{+} \cdots+c_{m} \delta_{m}^{+}$. Рассмотрим сумму

$$
S=\sum_{c_{1}, \ldots, c_{m} \in\{0,1\}} \operatorname{wt}\left(\delta^{+}\left(c_{1}, \ldots, c_{m}\right)\right) \text {. }
$$

Каждое слагаемое в $S$, кроме слагаемого, соответствующего нулевому набору, равно $2^{h}$ по лемме 31. Обозначим через $r$ число строк среди верхних $2^{2 h-1}+2^{h-1}$ строк матрицы $M$, содержащих хотя бы одну единицу в правой части матрицы $M$. Заметим, что если строка в верхней части матрицы $M$ содержит хотя бы одну такую единицу, то в точности $2^{m-1}$ из $2^{m}-1$ ненулевых наборов $\delta^{+}\left(c_{1}, \ldots, c_{3}\right)$ имеют единицу в этой строке. Поэтому $S=2^{h}\left(2^{m}-1\right)=r 2^{m-1}$. Отсюда $r=2^{h+1}-2^{h-m+1}$. В силу целочисленности $r$ справедливо неравенство $m \leqslant h+1$, что и требовалось доказать.

Утверждение теоремы 3 немедленно следует из структуры матрицы $M$ и леммы 32 . При $h=2$ оценка теоремы 3 не достигается. Осмелимся выдвинуть гипотезу.

Гипотеза. Для любого натурального $h$ максимально возможный аффинный ранг платовидной функции с носителем спектра мощности $4^{h}$ равен $2^{h+1}-2$.

\section{Список литературы}

1. Кузнецов Ю. В., О носителях платовидных функций. В сб.: Материалы VIII Международного семинара "Дискретная математика и ее приложения». МГУ, Москва, 2004, с. 424-426.

2. Логачев О. А., Сальников А. А., Ященко В. В., Булевы функции в теории кодирования и криптографии. МЦНМО, Москва, 2004.

3. Таранников Ю. В., О корреляционно-иммунных и устойчивых булевых функциях. Матем. вопросы кибернетики (2002) 11, 91-148.

4. Таранников Ю. В., О платовидных устойчивых функциях. В сб.: Материалы VIII Междупародного семинара "Дискретная математика и ее приложения». МГУ, Москва, 2004, с. 431-435.

5. Carlet C., Charpin P., Cubic Boolean functions with highest resiliency. In: Proc. 2004 IEEE Intern. Symposium on Inform. Theory, 2004, p. 497.

6. Carlet C., Sarkar P., Spectral domain analysis of correlation immune and resilient Boolean functions. Finite Fields Appl. (2002) 8, 120-130.

7. Kasami T., Tokura N., Azumi S., On the weight enumeration of weights less than $2.5 d$ of ReedMuller codes. Information and Control (1976) 30 (4), 380-395.

8. Pei D., Qin W., The correlation of a Boolean function with its variables. Lecture Notes Computer Sci. (2000) 1977, 1-8.

9. Zheng Y., Zhang X.-M., Plateaued functions. Lecture Notes Computer Sci. (1999) 1726, 284-300. 